http://doi.org/10.15359/ree.12-Ext.14

\title{
LA PARTICIPACIÓN EN EL AULA ESCOLAR RURAL: UN RETO PARA LA TRANSFORMACIÓN
}

\author{
Enid Arguedas Leitón ${ }^{1}$ \\ Docente de la Escuela Santa Marta de Siquirres \\ Limón, Costa Rica \\ Lidieth Núñez Castro ${ }^{2}$ \\ Docente de la Escuela de Pital \\ San Carlos, Costa Rica \\ Rodrigo Torres Hernández ${ }^{3}$ \\ Docente de la Escuela de Chase \\ Talamanca, Limón. \\ Ana Cecilia Vásquez Carvajal ${ }^{4}$ \\ Docente de la Escuela Ujarrás y Escuela San Vicente \\ Zona Sur, Costa Rica \\ Claudio Antonio Vargas Fallas 5 \\ Docente de la División de Educación Rural, CIDE-Universidad Nacional \\ Heredia, Costa Rica
}

Recibido: 11 de setiembre 2007 • Aprobado: 20 de noviembre 2007

Resumen: Este trabajo analiza el tema de la participación como un medio de ejercer el derecho a la educación, como forma de democratizar el proceso educativo y como vía para lograr pertinencia en el proceso. Se asume, que los padres, madres, estudiantes y otros miembros de la comunidad deben estar cerca de la escuela y aula, no sólo para colaborar con las tareas que tradicionalmente se les asignan a estas personas, sino para desarrollar una nueva forma de participación, que involucre la construcción de los procesos educativos. Para lograr este propósito, los autores y autoras enuncian una serie de estrategias útiles para el acercamiento entre escuela y comunidad.

Palabras clave: Participación, comunidad rural, escuela rural.

Abstract: This work analyses the participation as a way to exert the right to education, as a mean to democratize the learning process and as a channel to achieve pertinence in the educational process. It assumes that parents, students and other members of the community have to be near the school and the classroom not only to collaborate with the tasks traditionally assigned to them but also to develop a new way of participation that involves a construction process of the learning process. To achieve this purpose, the authors enunciate different strategies, useful to the get school and community together.

Key words: Participation, rural community, rural school.

\footnotetext{
Graduada de la primera promoción de la Maestría en Educación Rural Centroamericana. Labora en la Escuela Santa Marta de Siquirres, Limón, Costa Rica. Correo electrónico: enarlei@yahoo.com

2 Graduada de la primera promoción de la Maestría en Educación Rural Centroamericana. Docente de la Escuela de Pital, San Carlos, Costa Rica. Correo electrónico: lidygas@hotmail.com

3 Graduado de la primera promoción de la Maestría en Educación Rural Centroamericana. Docente de la Escuela de Chase, Talamanca, Limón. Correo electrónico: rotoher@yahoo.es

$4 \quad$ Graduada de la primera promoción de la Maestría en Educación Rural Centroamericana. Docente de la Escuela Ujarrás y Escuela San Vicente, ambas de la Zona Sur de Costa Rica. Correo electrónico: famave2002@costarricense.cr

5 Graduado de la primera promoción de la Maestría en Educación Rural Centroamericana. Docente de la División de Educación Rural de la Universidad Nacional y de la Facultad de Educación de la Universidad de Costa Rica. Correo electrónico: v.claudio.v@gmail.com.
} 
El campesino cree en la escuela espera mucho de ella, ve en ella la oportunidad de abrir las compuertas para la vida de sus hijos, pero a la postre no confía o confía poco en la escuela. La siente necesaria, pero lejana, idealmente atractiva pero esquiva, la mira con orgullo desde sus hijos y a la par no percibe con frecuencia su utilidad práctica.

Juan Bautista Arrien (2003)

\section{INTRODUCCIÓN}

En el paradigma tradicional de la educación, la y el docente ocupan un lugar central en el desarrollo del proceso educativo. Tanto la planificación como la ejecución de la mediación pedagógica y la labor administrativa, son orientadas por el educador y la educadora con una mínima participación de otros actores de la comunidad escolar.

Dentro de esta perspectiva poco democrática de la educación, la participación de padres y madres se limita a tareas asistenciales y a una actitud pasiva y dependiente de las y los estudiantes.

Para Crespo, citado por (van der Bijl, 2003): "El maestro rural mantiene la idea de que la participación de los padres y madres de familia es aceptable, cuando se trata de prestar las manos, para arreglar la escuela o conseguir alguna obra. Los padres y madres, por su parte, han sustentado su distancia con la escuela en la opinión de que para la enseñanza está el maestro" (p. 28).

Lejos de considerarse aliados en la educación de los menores, las relaciones entre docentes, padres y madres de familia se han tornado, en algunos casos, conflictivas. Como si estuvieran ubicados en aceras diferentes, unos responsabilizan a los otros del fracaso escolar de las y los menores.

La legislación vigente, que enfatiza los derechos de niños, niñas y adolescentes, así como la creación de instituciones como la Sala IV y la Defensoría de los Habitantes ha dado poder a los padres y madres para reclamar por sus derechos y los de sus hijos e hijas. Sin embargo, este empoderamiento aún no trasciende a lo cooperativo o colaborativo. Es decir, hasta ahora, la comunidad sabe que puede exigir una educación de calidad, pero aún no es consciente de que también puede y debe ser co-educadora en los procesos de aprendizaje.

Como resultado, la educación que se da desde la escuela se encuentra desvinculada de su entorno, no sólo porque se basa en un currículo que no responde a lo que les interesa a los niños y niñas de la comunidad, o a lo que éstos necesitan, sino también porque desaprovecha a la misma comunidad como recurso para desarrollar el currículo. "La escuela se ha aislado de su entorno, se ha convertido en un mundo cerrado y protegido, separado de la vida de todos los días y no juega ningún papel real en la comunidad" (van der Bijl, 2003, p. 29).

Desde una perspectiva renovadora de la educación, se concibe la participación como un hecho mucho más complejo, que exige responsabilidad y compromiso de todos los actores, y que está fundamentado en una relación de equidad, con un alto sentido de pertenencia e identificación con el grupo. La participación adquiere sentido cuando se constituye en un medio para la construcción de un proyecto común en el que se requiere del aporte de todos y todas. No es un fin en sí mismo, 
es un medio para lograr cambios cualitativos en los niveles personal y colectivo.

Una práctica pedagógica participativa nace desde la realidad de los actores del proceso educativo, de sus necesidades, fortalezas y aspiraciones, adquiriendo significado para todos sus miembros. "Cuando se ha decidido hacer educación rural, desde dentro, desde la gente campesina, han surgido iniciativas muy beneficiosas para la educación de los sectores rurales” (Arrien, 2003, p. 23).

Fuera de la institución escolar, se encuentran realidades que tienen que enfrentarse día a día. Por lo tanto, la escuela tiene que ser un espacio mediador, donde se pueden encontrar las respuestas a esas realidades. Es necesario que el o la docente cuente con el apoyo de los vecinos, los padres y madres de familia y los y las líderes comunales, para que compartan su bagaje cultural y contribuyan a ampliar las nociones que los estudiantes poseen. De esta manera, se trata de encontrar un vínculo y un equilibrio entre lo local y lo universal, lo personal y lo colectivo, lo tradicional y lo nuevo, la comunidad y el aula escolar.

Además, este proceso participativo permite el fortalecimiento de las relaciones afectivas, el enriquecimiento mutuo y el sentido de arraigo comunitario. La escuela deja de ser una isla y se integra como elemento vital dentro de la comunidad. Nutre a la comunidad pero también se nutre de ella.

Se transita, entonces, de un papel de espectador a uno de actor. Se abre el espacio para que aquel que estaba silenciado, pueda hablar, aquel que estaba excluido de la toma de decisiones, pueda decidir, o aquel que solo habla, se comprometa a trabajar. Sin embargo, no basta con el deseo de querer subirse al escenario. Tanto padres y madres, como estudiantes y docentes deben aprender cómo asumir este nuevo papel. Es aquí donde el o la docente, como líder, debe crear las posibilidades para que los otros actores se animen a subirse al escenario, y luego, para que actúen. Se trata de montar una obra sobre un guión no escrito, de la cual solo se tiene el esbozo. Se trata de crear en el camino. ¿Quién enseña a quién cómo participar? En este caso, tendría que afirmarse, como Freire, nadie educa a nadie, la verdadera educación es diálogo. Entonces, es al docente a quien le corresponde, aunque no de manera exclusiva, propiciar este encuentro dialógico.

Cuando la comunidad logra empoderarse, se abre la posibilidad de innovar, es decir, de introducir cambios desde las bases mismas de la cotidianidad. Así, cada escuela puede construir su propia propuesta pedagógica y, por lo tanto, superar el currículo estandarizado. Estos cambios hacen que la escuela adquiera un nuevo significado para los estudiantes, porque se integra con la realidad cotidiana, ya no está separada de ella.

En síntesis, dentro de un nuevo paradigma de la educación rural, nace la comunidad de aprendizaje como un todo donde se integran activamente personal docente y administrativo, estudiantes y comunidad. En un proceso de diálogo, se crean espacios para que todos aporten en la construcción de un proyecto pedagógico consensuado vinculado estrechamente con el desarrollo de la comunidad. La renovación de la escuela rural exige, necesariamente, la participación de la comunidad en forma conciente, activa y permanente.

\section{EL Y LA DOCENTE EN EL AULA ESCOLAR}

El aula escolar debe entenderse como un espacio más amplio que el edificio escolar. En este sentido, todo espacio donde se pueda aprender se constituye en aula escolar. La huerta, la chanche$r a$, la finca, la montaña, el río, la playa, son espacios con infinidad de recursos que los convierten en fuentes vitales de aprendizaje. Esta percepción amplia del espacio de aprendizaje, implica, además, reconocer que todos y todas las personas que se ubican en él son también coaprendices e incorpora 
a nuevos participantes en la escena educativa.

En esa aula, tan amplia como el mundo, la comunidad debe tener la participación para seleccionar los contenidos culturales y encontrar, con la guía del o la profesional en educación, los procesos metodológicos más adecuados para aprenderlos.

El docente y la docente son, sin duda, los principales gestores de la promoción de la participación en los procesos de aprendizaje, pues tienen la capacidad de provocar o limitar la participación de los y las estudiantes y de los padres y madres de familia; de él y ella depende que se dé una participación real o conformarse con una participación tradicional, centralizada y vertical.

Mas ¿cómo lograr más y mejores niveles de participación dentro del aula escolar? Por un lado, hay que reconocer que el docente rural se encuentra muchas veces abandonado y con poco apoyo del Estado, lo que afecta su motivación y limita ampliamente su capacidad de trabajo. Por otro, no todos en la comunidad están interesados en participar; sino que, ocupados en sus propios asuntos, delegan en otras personas este derecho.

Además, debe existir una política educativa que fortalezca la autoestima del y la docente rural mediante reconocimiento social y económico. Junto a estos elementos, el y la docente deben poseer herramientas cognoscitivas, destrezas y habilidades que les permitan enfrentar los desafíos y retos que exige la realidad rural comunitaria y escolar.

Por ello, es importante que los docentes rurales tengan un perfil de formación apropiado, que además de su actitud, disponibilidad y compromiso, incluya aspectos como:

- Conocimientos sobre el concepto y visión de la ruralidad.

- Pedagogía rural y diseño curricular.

- Manejo de procesos de educación no formal.

- Uso de la metodología de investigación-acción.

- Conocimientos sobre promoción, liderazgo y participación comunitaria.

- Conocimientos de técnicas de conducción y animación grupal, aptas para diferentes edades.

- Capacidad para diseñar, ejecutar, evaluar y sistematizar proyectos.

- Capacidad para valorar, rescatar, sistematizar y aprovechar la cultura popular y dentro de ella, el conocimiento popular.

Esto quiere decir que se requiere un docente con los siguientes rasgos:

- Facilitador de procesos, capaz de atraer e incorporar actores/as a la escena pedagógica.

- Gran motivador, capaz de propiciar la aprehensión del conocimiento y el crecimiento intelectual a través de técnicas y métodos de mediación que induzcan a los y las estudiantes a ver, en su entorno, el primer recurso de aprendizaje.

- $\quad$ Con una nueva actitud que demande creatividad, iniciativa y dinamismo, cualidades que enriquecen los procesos de mediación y de técnicas para el trabajo grupal y colectivo.

- Hablante y escribiente de un bilingüismo cultural, es decir, de dos lenguajes: su lenguaje y el de la comunidad; este último con sus propios valores, su espiritualidad, su uso del idioma, sus costumbres diarias, con un respeto profundo y una valoración de lo que el otro significa, como lo afirma Durston (1997).

- Humilde para reconocer que no es dueño/a absoluto del conocimiento sino que, también es un aprendiz que se enriquece tanto de los aportes de los estudiantes como de los padres de 
familia y la comunidad.

- $\quad$ Abierto al diálogo, observador perspicaz e investigador permanente, capaz de aprovechar los recursos que el medio ofrece para el currículo escolar.

- Que inspire confianza, seguridad, entusiasmo y alegría para lograr una participación activa y permanente.

- $\quad$ Participante activo dentro de la comunidad.

Si hablamos de participación en el aula escolar, se debe considerar la existencia de una política institucional que oriente el quehacer de los actores/as del proceso educativo, y un liderazgo abierto y democrático que permita procesos participativos dentro del aula. La participación que logre el o la docente debe ser parte de una estrategia institucional, que si bien es cierto no es una camisa de fuerza, debe servir de apoyo a las iniciativas que nazcan en su interior.

La descentralización y la autonomía son principios de la participación que deben ponerse en práctica desde los espacios micros del sistema educativo, que en este caso, está constituido por el aula escolar. No podrían garantizarse verdaderos procesos participativos dentro de una estructura administrativa tradicional, vertical y centralizada.

El y la docente deben crear los espacios para la construcción común del proceso educativo a través de una actitud de diálogo y escucha permanente.

\section{ALGUNAS ESTRATEGIAS QUE PUEDEN SER ÚTILES}

\section{Sensibilización}

El y la docente han de iniciar un proceso de inducción que fortalezca en los padres de familia la capacidad de asumirse como actores/as del aprendizaje de sus hijos e hijas, sobre todo de quienes consideran que la educación solamente es responsabilidad de la escuela. Para ello, debe abrir espacios de discusión para conocer lo que los padres, madres y estudiantes piensan sobre lo que se debe aprender, cómo aprenderlo y con qué recursos; y debe ofrecer la oportunidad para que expresen sus sueños, ideales y metas.

\section{Profundizar en el conocimiento de la comunidad}

El y la docente, junto con los padres, madres y la comunidad, deben realizar un diagnóstico participativo que permitirá, entre otras cosas, valorar las fortalezas y debilidades, en los ámbitos individual y colectivo, que pueden incidir en el aprendizaje de los niños y las niñas. Por otro lado, el diagnóstico dará los insumos para determinar lo que los niños y las niñas necesitan de la institución, de manera que esto sea incluido en el planeamiento didáctico.

Además, el diagnóstico dará la oportunidad para que todos digan qué pueden aportar al proceso educativo desde su condición de estudiante, padre, madre o vecino.

\section{Planificación del programa escolar}

Elaboración de una propuesta programática del currículo escolar, tomando en cuenta la información 
aportada por los actores/as participantes, que incluye fines, objetivos, metas, recursos y actividades. Ejecución de los procesos de aprendizaje

Hacer un inventario de los recursos con que cuentan los padres, madres y estudiantes y que pueden ser aprovechados para el desarrollo de las clases. En estos recursos, se toman en cuenta las habilidades, destrezas, conocimientos y oficios de los miembros de la comunidad, así como los recursos materiales.

\section{Realización de proyectos de educación no formal}

Es importante establecer un contacto continuo entre el padre y la madre de familia, el maestro o la maestra y la comunidad, para realizar actividades de educación no formal que respondan a inquietudes de los mismos padres, madres y estudiantes. Estas actividades pueden asumir muchas formas, entre ellas la celebración de efemérides, la organización de eventos sociales, la confección de materiales, la atención de problemas internos al grupo y otros.

\section{Modelo pedagógico basado en proyectos o unidades integradas}

El docente debe ofrecer espacios de participación a los niños y niñas mediante un modelo pedagógico que favorezca el aprendizaje por medio de proyectos o unidades integradas. Este modelo permiten la puesta en práctica de un currículo integrado, superando la segmentación del conocimiento en asignaturas, a la vez que permite la realización de actividades vinculadas con lo que al niño y a la niña le interesa verdaderamente y una proyección institucional hacia la comunidad.

\section{La interculturalidad como recurso para el aprendizaje}

Las escuelas, y en particular las aulas, son espacios muy ricos por su diversidad cultural. Dentro de la misma aula existe la multiculturalidad, dado que cada niño y cada familia representan diferentes formas de aprender y vivir. En las instituciones se deben tomar en cuenta no solo los conocimientos previos, sino también las diferencias que los educandos tienen, pues éstos son fuente para enriquecer los procesos de aprendizaje, evitar las exclusiones y trascender hacia la interculturalidad.

\section{Aprovechamiento de las estructuras organizativa existente}

En cada grupo escolar, hay una junta directiva de padres y madres de familia, una directiva de sección y algunos comités integrados por estudiantes. Estos organismos pueden desempeñar un nuevo rol al asumir actividades en el diseño del programa escolar, la organización de actividades escolares o extraescolares, como giras, expediciones, confección de materiales, actividades deportivas y artísticas, atención de necesidades especiales dentro del 
aula y muchas otras más.

\section{CONSIDERACIONES FINALES}

La educación debe ser un proceso democrático, no sólo en la elaboración de sus fundamentos pedagógicos, sino también en la definición de sus fines y en la construcción de su currículo. También, debe serlo en la concreción del hecho educativo. La única manera de lograr que se viva este proceso democratizador de la educación, es mediante la participación equitativa de todos los actores/as que intervienen en el proceso educativo. Esto incluye por supuesto, como lo afirma Freire (1994), al Estado y sus instituciones, pero también incluye a cada uno y cada una de las personas involucradas: educadores y educandos, padres y madres de familia, personal administrativo - cuando lo hay-, y los demás miembros de la comunidad, porque el proceso educativo también les incumbe.

Esta participación no ocurre al azar o por casualidad. Es el resultado de un proceso que debe ser intencional: ser provocado, orientado, estimulado y entrenado. Los miembros de la comunidad tienen este deber, y también lo tiene la escuela, en su papel de conductora de procesos educativos. El personal de la escuela en conjunto con la comunidad, debe buscar estrategias y situaciones en las que se pueda promover la participación, para educar en la participación para ejercitarla, para crear convencimiento sobre sus beneficios y para construir el empoderamiento que le sucede.

La participación es el ejercicio democrático del poder, es decir, la igualdad de oportunidades en la toma de decisiones y en la ejecución de tareas. En este sentido, la participación como medio, permite la transformación no solo del aula escolar, particularmente el aula rural, y de la comunidad sino, de cada uno de los sujetos involucrados. Se trata, entonces, de una colectividad comprometida en la consecución de un fin común, que es el mejoramiento constante de su calidad de vida.

\section{REFERENCIAS}

Arrien, J. B. (2003). La educación Rural en Centroamérica (Conferencia Inaugural). En M. E. Aguilar y Cerdas (Comp.), La educación rural en Centroamérica. II Seminario centroamericano de educación rural (pp. 1-23). Heredia: EUNA.

Durston, J. (1997). La participación comunitaria en la gestión de la escuela rural. En E. Cohen (Aut.), Educación, eficiencia y equidad (pp. 68-84). Santiago, Chile: Ediciones SUR.

Freire, P. (1994). Participación y educación Comunitaria [Extracto de Ponencia]. En: M. Castells (Aut.), Congreso Internacional de "Nuevas perspectivas críticas en educación” (pp. 1-5). Paidós, Barcelona: División de Ciencias de la Educación. Extraído el 22 de agosto de 2007 de http://peuma.unblog.fr/files/2007/12/educacionyparticipacion.pdf

van der Bijl, B. (2003). Escuela rural y desarrollo comunitario (2a ed.).Heredia, Costa Rica: Universidad Nacional. 